\title{
Special Issue on Discrete Geometry for Computer Imagery
}

\author{
Nicolas Normand $^{1}$ - Jeanpierre Guédon ${ }^{1}$ - David Coeurjolly ${ }^{2}$
}

Published online: 29 May 2017

(C) Springer Science+Business Media New York 2017

\section{Preface}

Digital geometry aims at defining proper geometric models and properties onto subsets of the integer plane/space $\left(\mathbb{Z}^{\mathrm{d}}\right)$. It also intends to define efficient algorithms for digital object topology and geometry processing. Digital geometry plays an essential role in the field of image analysis, computer graphics, pattern recognition, shape modeling, computer vision, and document analysis. Beside the fact that all data in the computer are unavoidably discrete, digital geometry is a subfield of discrete geometry in the sense that it focuses on geometrical objects defined on lattices and grids.

Even if the domain emerged during the second half of the twentieth century with the birth of computer graphics and digital image processing, many links have been demonstrated between digital geometry results and fundamental theorems in mathematics (arithmetic, geometry, topology...), discrete mathematics (word theory, combinatorics, graph theory...) or computer science (algorithmic, computational geometry, image processing, discrete tomography, computer graphics...).

The center of gravity of the international digital geometry community is mainly in Europe with couple of research groups from India, Japan and Australia and USA. In association with the International Association for Pattern Recognition (IAPR), the community interacts within the "Discrete Geometry and Mathematical Morphology" Technical Committee, TC18 (http://tc18.org). The TC18 website also gathers resources (datasets, source code, bibliography...) for interested readers.

In this special issue, selected articles cover many aspects of digital geometry: digital topology, digital shape modeling, digital curve and surface analysis, geometric and distance transform and mathematical morphology.

The guest editors would like to warmly acknowledge the reviewers for their invaluable contribution to this special issue.

\footnotetext{
David Coeurjolly

david.coeurjolly@liris.cnrs.fr

LS2N, Team IPI, Polytech Nantes, France

2 Université de Lyon, CNRS, LIRIS UMR 5205, Villeurbanne, France
} 